\title{
PERFORMANCE ANALYSIS OF LATVIAN ZENITH CAMERA
}

\author{
Ansis ZARIN̦Š ${ }^{*}$, Augusts RUBANS, Gunārs SILABRIEDIS \\ Institute of Geodesy and Geoinformatics, the University of Latvia, \\ Raina bulvāris 19, LV-1586, Rīga, Latvia
}

Received 24 January 2018; accepted 14 March 2018

\begin{abstract}
Since finalizing of design in 2016, the digital zenith camera of the University of Latvia was involved in a number of test observations as well as field observations at about 70 different sites. The paper presents analysis of observation results, estimation of instrument's performance and accuracy. Random and systematic error sources are outlined. Impact of anomalous refraction on vertical deflection determination is discussed. Results of adaptation of GAIA reference star catalog for astrometric data reduction are reported.
\end{abstract}

Keywords: digital zenith camera, geodetic astronomy, vertical deflections, geoid, anomalous refraction.

\section{Introduction}

Basically, design of digital zenith camera in the institute of Geodesy and Geoinformatics of the University of Latvia was concluded in 2016 (Zariņš, Rubans, \& Silabriedis, 2016). Up to the end of 2017 a number of test observations at a fixed test site, as well as field observations at about 70 different sites were performed. A number of further improvements of design, measurement methodology and control and data processing software were inspired in the process. Analysis of collected data allow to draw conclusions about properties of the instrument and measurement results.

\section{Observation methodology}

Unlike other known instruments of this type, our zenith camera can employ any amount of rotation between observation positions; actual orientation of it is determined by astrometric processing of star field images (Zariņš et al., 2016). As $180 \mathrm{dg}$ rotation offers the best definition of expected quasi-circular trajectory of ellipsoidal zenith projection on rotating instrument coordinate system, a regular observation session is made of a number of such position pairs. In order to represent whole range of spatial orientations, a smaller rotation (for $20-45 \mathrm{dg}$ ) is done between $180 \mathrm{dg}$ pairs, therefore complete session includes positions, distributed along all azimuths. Although such distribution is not strictly necessary for data processing purposes, we find it beneficial for detecting possible anomalies or errors in data.
Acquisition of a single image and associated tiltmeter data set (a frame) typically takes $8-10$ seconds. As rotation to the next position and subsequent leveling can take up to a minute, acquisition of several frames per position are convenient, improving definition of astrometric position. In situation, when dispersion of astrometric positions due to atmospheric turbulence is one of the main accuracy degrading factors, we find multiple frame tactics more efficient. At 10 frames per position, data acquisition together with rotation and leveling consumes about 2 minutes (4 minutes per position pair).

\section{Data model}

Besides random effects of atmospheric turbulence and microseismics, the measurement process is complicated also by factors of systematic nature. The most pronounced of them are variability in relative orientation of astrometric and leveling subsystems, caused, most probably by thermal deformations in construction elements of the instrument. Another major effect is caused by anomalous refraction, leading to variations in the measured value of vertical deflection itself. Both effects to some extent can be distinguished by the tendency of thermal drift to generally retain continuity in instrument's coordinate system (which is rotating in the process of measurements), while variations of anomalous refraction should similarly retain continuity in Earth-connected coordinate system. If the rotating instrument's coordinate system is used in

*Corresponding author. E-mail: ansiszx@inbox.lv 
calculations, at least in limited time intervals thermal drift can be approximated by model (1). It describes dependence of projections $X, Y$ of ellipsoidal zenith to CCD image on image orientation azimuth $\mathrm{A}$ and time dT:

$$
\begin{gathered}
X=x_{0}-\sin A \times \xi+\cos A \times \eta+d T \times \dot{X}, \\
Y=y_{0}+\sin A \times \eta+\cos A \times \xi+d T \times \dot{Y} .
\end{gathered}
$$

Here $X, Y$ are already corrected for residual tilt according to (1) in (Zariņš et al., 2016); $\xi$ and $\eta$ are North-South and East-West components of vertical deflection; $\dot{X}$ and $\dot{Y}$ represent drift speed components.

As in reality the drift is not a linear function of time, accuracy of model (1) deteriorates with increasing of represented time interval.

Although variations of vertical deflection value can be introduced in model (1) as well, attempts to do so using similar linear time dependency did no lead to conclusive

a)

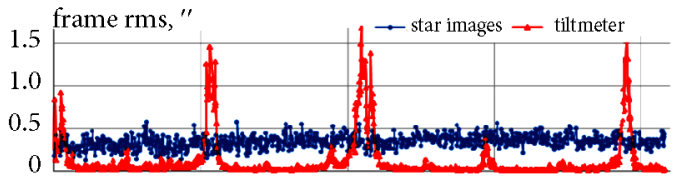

b) 0.6 model rms,"

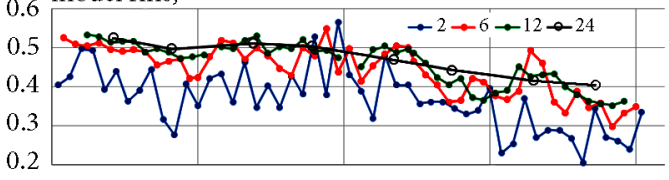

c)

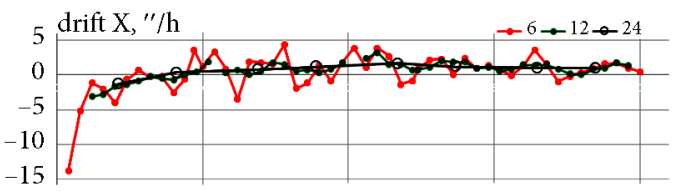

d)

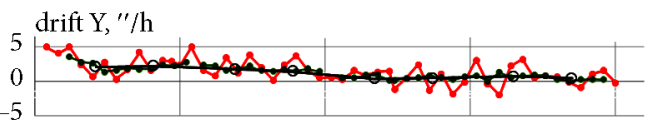

e)

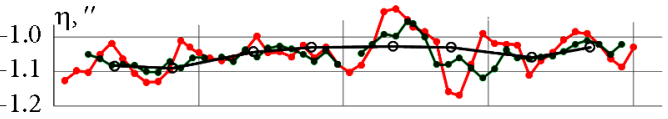

f) 5

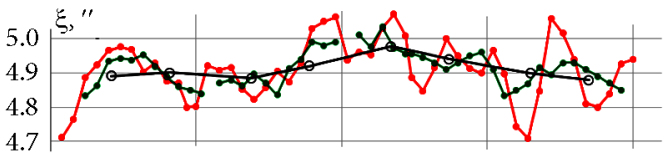

g)

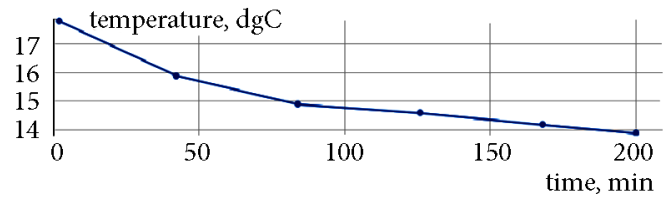

Figure 1. Results of sample session as function of time: a) star position and tiltmeter reading rms for a frame; b) model approximation rms for different data windows; c) drift $X$ component evaluation; d) drift $Y$ component evaluation; e) vertical deflection $\eta$ component evaluation; f) vertical defection $\xi$ component evaluation; e) ambient temperature. Size of data window (number of positions) shown by different colors for b) to $\mathrm{f}$ ) results - probably, due to more complicated nature of this variability and shorter linearity time.

As a result of above variabilities, increase of the number of observed positions (represented time interval) makes model approximation residual rms gradually bigger; obtained model parameter values are averaged. Analysis of a number of observation sessions indicate, that a minimum of about 3 pairs of positions (about 10-12 minutes of time) are needed to reduce effect of random dispersion sources enough for reasonable drift and vertical deflection evaluation. This seems also to be the shortest time period, for which accuracy of vertical deflection determination is enough to notice effects of anomalous refraction (systematic changes of vertical deflection value over time). Consequently, short sessions with small modeling errors do not have enough data points to average out random fluctuations, mainly of atmospheric turbulence origin. Therefore, some compromise session duration have to be used. Besides, in any case observations should be carried out as fast as possible to minimize impact of thermal drift and maximize time resolution.

\section{Data analysis}

In order to evaluate random dispersion, thermal drift and identify possible anomalous refraction effects, observation data for a number of sessions was processed using moving time interval windows of 4 sizes: $2,6,12$ and 24 positions (containing 10 frames each), corresponding to time intervals of 4, 12, 24 and 48 minutes. For each window parameters of approximating model (1) were calculated.

In general, properties of the results for all sessions were similar, differing mainly in parameter values due to diverse atmospheric conditions, temperature variances, wind and microseismics levels. Analysis of results hereafter is provided on an example of one of the longest uninterrupted sessions (June 17, 2017), consisting of 1050 frames in 106 positions, over time interval of 3.5 hours. Graphical representation of results is given on Figures 1, 2 and 3.

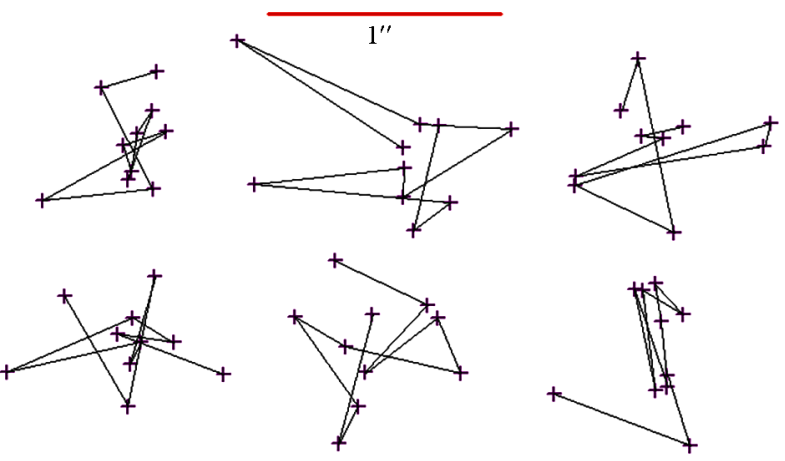

Figure 2. Position of corrected zenith point on CCD (orientation arbitrary) for 6 consecutive positions around minute 180 of sample session. Consecutive frames connected by line 


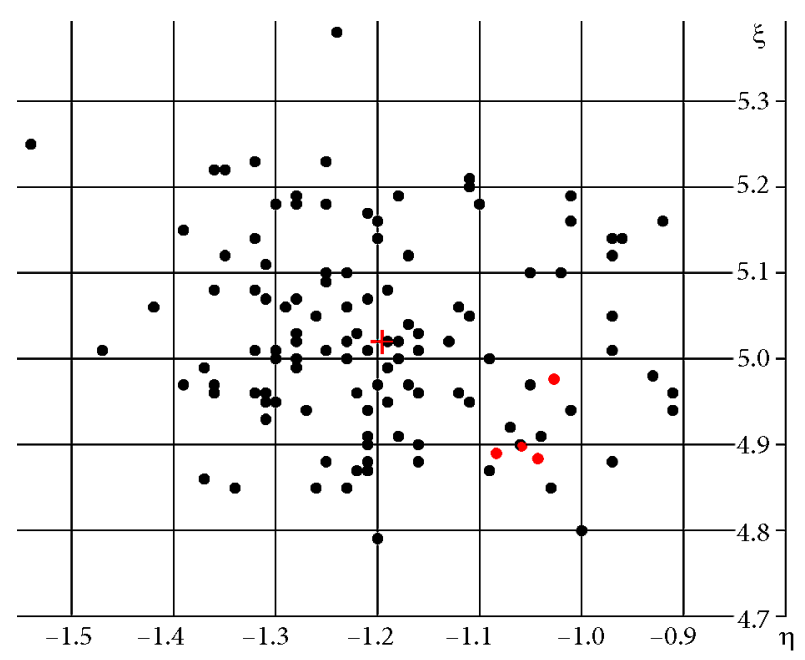

Figure 3. Vertical deflection determination results at the test site within a year. Each point represent averaged result of about 40 minutes long session

\subsection{Frame}

Frame data consists of a star field image and a set of tiltmeter measurements, made during exposure and a few seconds before and after it. The sample session data contained 30-50 star images and 30-60 tiltmeter readings per frame. Star image CCD coordinates were fitted to respective coordinates in reference star catalog using least squares approximation by 6-parameter transformation model; CCD coordinates of astrometric zenith point for the mid-exposure time moment were calculated. Tiltmeter measurements were approximated using linear time dependency; residual tilt for the mid-exposure time moment was calculated - to be applied as correction to zenith point CCD coordinates according to formulas (1) in (Zariņš et al., 2016).

Atmospheric turbulence effect on star images together with discrete nature of images (with pixel size close to $1 "$ ) seem to be dominating dispersion sources for astrometric model. Star residual rms (Figure 1a, blue) is typically between $0.2^{\prime \prime}$ and $0.6^{\prime \prime}$ (see also Figure 4), can be slightly changing during the session, both increasing (as in the sample session), or decreasing. Reasons of this changeability are not apparent, probably changes in atmosphere properties might be involved.

Microseismics level (Figure 1a, red) generally tend to be below a few tens of milliarcseconds, except some cases of nearby heavy transport influence (flashes on Figure 1a, for instance, are caused by passing of trains along $0.5 \mathrm{~km}$ distant railway line). Even then, microseismics are averaged over the frame duration and practically no effect of microseismics flashes on star and model approximation rms can be found.

At such tiltmeter readings and individual star rms levels, and at 30-50 stars per frame, estimated zenith point coordinate dispersion should be well below 0.1 ". In reality, however, frames taken at a position each 8 seconds, show much bigger scattering of corrected zenith positions, than could be expected at sub-0.1" dispersion (Figure 2). Dispersion rms for 10 frames of a position typically is $0.2^{\prime \prime}$ to $0.5^{\prime \prime}$, amplitude of scattering can exceed 1". Generally, the nature of scattering is quite random, although some back-and-forth patterns can be noticed, suggesting presence of oscillatory components with periods in seconds to tens of seconds. Presumably, origin of this scattering lays in the same atmospheric fluctuations, as responsible for image scintillation, albeit of lower frequencies, bigger spatial scale and smaller amplitude of image displacement. At still bigger temporal and spatial scale that same effect is known as anomalous refraction.

\subsection{Model}

As it should be expected, model approximation rms (Figure $1 \mathrm{~b}$ ) for the shortest 2-position (4 minutes) window is the smallest, with little drift and anomalous refraction effects to accommodate. Model residual values for it are close to single position case (as on Figure 2).

Model approximation accuracy deteriorates with increase of window duration, especially if variations of thermal drift or anomalous refraction are present. Thermal drift influence is dominating, for amplitude of anomalous refraction effect is limited to a few tenths of arcsecond and only slightly influences model approximation accuracy in comparison with thermal drift variability effects, easily exceeding arcsecond at the beginning of sessions, when cooling down of the instrument still continues.

In majority of sessions model approximation rms noticeably decreases towards the end of session. We attribute this effect mainly to decrease of thermal expansion effects with more thorough adapting of the instrument to environment temperature and slower environment temperature change later in night. Possibly, also atmospheric turbulence somewhat calms down, making variations of refraction smaller. Such effect is sometimes noticed in frame star image residuals (but not always - in sample session, for instance, star rms is rather increasing towards the end of session (Figure 1b), possibly indicating difference in behavior of high and low frequency components of turbulence).

\subsection{Drift}

As anticipated, short duration data window does not provide reliable thermal drift estimation. Two position (4 minutes) drift estimates are very inconsistent, with dispersion significantly exceeding average value. Apparent source of such instability seems to be abovementioned dispersion of frame zenith projections within a position $0.5^{\prime \prime}$ variability within 2 minutes from position to position means $15 \mathrm{l} / \mathrm{h}$ random component in drift calculations; 2-position drift variations of such amplitude are common. Random component for 6-position (12 minutes) data window is much smaller (Figure 1c and d), but still noticeable; for 12-position (24 minutes) window systematic trends already dominate over random component. 24-position (48 minutes) window is clearly over-averaged. Consequently, 
some structure of drift can be resolved starting with 10-20 minute temporal resolution. Drift speed variations with characteristic periods from tens of minutes to about one hour are surely present. Common trend is big drift values at the start of session, especially early in the night, when environment rapidly cools down, or if instrument is not properly cooled before session. With time, drift rapidly decreases, finally stabilizes at some value (typically a few arcseconds per hour), depending on ambient temperature behavior.

\subsection{Vertical deflection components}

Alike drift, also vertical deflection component estimation (Figure 1e and $\mathrm{f}$ ) is not consistent for 2-position data window, possessing random component of the same amplitude as for frame zenith projections within a position - up to about 0.5 ". 6-position (12 minutes) solutions as well retain some random component, although presence of semi-systematic changes of longer periods (probably mostly of anomalous refraction origin, however some instrumental effects cannot be completely ruled out) is apparent. For longer duration data windows semisystematic variability component dominates or is overaveraged. Consequently, same as for drift, some structure of vertical deflection component dependence on time can be resolved starting with 10-20 minute temporal resolution. Such variability so far has been found in practically all measurement sessions with sufficient duration. If the target is acquisition of true vertical deflection values, presence of variability in measurement results (whatever it's cause) imposes limitations to accuracy - in an observation session of limited duration we get value representing only that duration. Our present impression is, that for acquisition of momentary value of vertical deflection duration of data window is optimal at 20-30 minutes; accuracy of such result can reach $0.05 "$. However, longer sessions (possibly, many sessions, distributed over time interval, spanning several years) are necessary to estimate temporal properties of measured values; momentary value can deviate from the true value for as much as $0.1^{\prime \prime}-0.3^{\prime \prime}$, as observations at a test site over a year demonstrate (Figure 3). For instance, the sample session's results (red points on Figure 3) are offset for about 0.15 " from the average value for the test site (red cross).

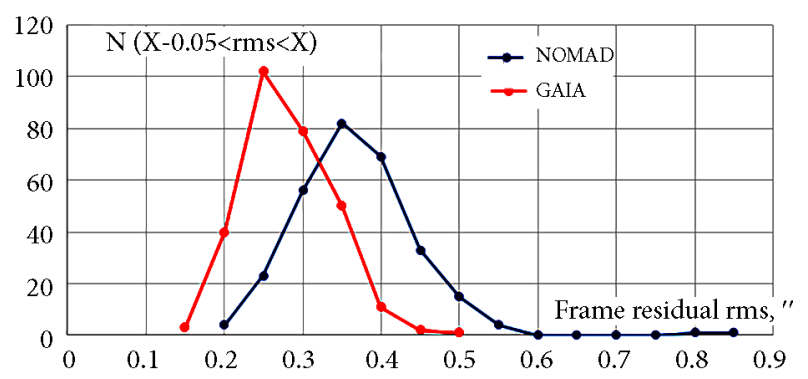

Figure 4. Distribution of reference star residual $\mathrm{rms}$ for a session of 300 frames - comparison of GAIA and NOMAD catalogs

\subsection{Anomalous refraction}

Variations of vertical deflection value, found in zenith camera measurements, with high probability can be attributed to anomalous refraction effects (Hirt, 2006; Hirt \& Seeber, 2008; Hirt, Bürki, Somieski, \& Seeber, 2010). Contribution of anomalous refraction is present in practically every measurement set, adding some offset from true vertical deflection value to results for sessions of limited duration. As Figure 1e and $\mathrm{f}$ indicate, variability of this offset can reach $0.15^{\prime \prime}$ even during a few hours; in (Hirt, 2006) very similar properties of variability are found.

The obvious presence of relatively short-period (tens of minutes) vertical deflection variations might suggest responsibility of a local mechanism - like topography interaction with low-altitude atmospheric processes. Attempts to correlate these variations to larger scale (tens to hundreds of kilometers) atmospheric pressure gradient did not show conclusive results. However, possibility of long-period (days or more, up to seasonal or even semipermanent) anomalous refraction effects cannot be ruled out. More thorough long-term observations in several test locations might give better insight in this issue. Probably, simultaneous observations with several adjacent instruments would be an efficient method to distinguish instrument-attributed variations from changes in measured quantity itself and find the spatial properties of anomalous refraction effects.

\section{GAIA catalog}

In September 2016 the first release of GAIA astrometric reference star catalog was published (Lindegren et al., 2016). It includes astrometric positions for majority of stars up to magnitude 20 with a few milliarcsecond accuracy (better than milliarcsecond for magnitude $<11.5$ ), offering substantially improved performance in astrometric calculations. A subset of GAIA catalog was adapted for use in zenith camera data processing. As anticipated, comparison with previously used NOMAD (consisting, for our purposes, mainly of USNO-B data of magnitude up to 14) showed improvement in rms of star position residuals in a frame for about $0.1 "$ (Figure 4). In addition, the long tail of distribution, present for NOMAD, is no more there for GAIA, suggesting presence of considerable number of inaccurate data in NOMAD. Besides, systematic differences for up to $0.15^{\prime \prime}$, depending on right ascension of zenith point and, apparently, reflecting systematic older catalog errors, were found. Hence we now use GAIA and have recalculated all old data with it.

\section{Conclusions}

Analysis of zenith camera observations in the test site as well as a number of field observations indicated good accordance to expected operational and accuracy properties of the instrument. Adaptation of the new GAIA reference star catalog and a number of hardware, software and 
methodology adjustments were done in the process of observation campaign, considerably improving performance and ease of use. The camera is now involved in regular observations in behalf of a regional geoid parameter improvement project.

Atmospheric turbulence and anomalous refraction effects are found to be the most significant factors, limiting accuracy. Quantitative properties of them are closely resembling results, found in earlier zenith camera research projects (Hirt \& Seeber, 2008; Hirt et al., 2010). Long-term observations in several test sites and simultaneous observations with several adjacent zenith cameras might yield better insight in the anomalous refraction problem.

\section{Funding}

This work was supported by the European Regional Development Fund under Grant No 1.1.1.1./16/A/160.

\section{References}

Hirt, C. (2006). Monitoring and analysis of anomalous refraction using a digital zenith camera system. Astronomy and Astrophysics, 459, 283-290. https://doi.org/10.1051/0004-6361:20065485

Hirt, C., Bürki, B., Somieski, A., \& Seeber, G. (2010). Modern determination of vertical deflections using digital zenith cameras. Journal of Surveying Engineering, 136(1), 1-12. https://doi.org/10.1061/(ASCE)SU.1943-5428.0000009
Hirt, C., \& Seeber, G. (2008). Accuracy analysis of vertical deflection data observed with the Hannover digital zenith camera system TZK2-D. Journal of Geodesy, 82(6), 347-356. https://doi.org/10.1007/s00190-007-0184-7

Lindegren, L., Lammers, U., Bastian, U., Hernández, J., Klioner, S., Hobbs, D., Bombrun, A., Michalik, D., Ramos-Lerate, M., Butkevich, A., Comoretto, G., Joliet, E., Holl, B., Hutton, A., Parsons, P., Steidelmüller, H., Abbas, U., Altmann, M., Andrei, A., Anton, S., Bach, N., Barache, C., Becciani, U., Berthier, J., Bianchi, L., Biermann, M., Bouquillon, S., Bourda, G., Brüsemeister, T., Bucciarelli, B., Busonero, D., Carlucci, T., Castañeda, J., Charlot, P., Clotet, M., Crosta, M., Davidson, M., de Felice, F., Drimmel, R., Fabricius, C., Fienga, A., Figueras, F., Fraile, E., Gai, M., Garralda, N., Geyer, R., González-Vidal, J. J., Guerra, R., Hambly, N. C., Hauser, M., Jordan, S., Lattanzi, M. G., Lenhardt, H., Liao, S., Löffler, W., McMillan, P. J., Mignard, F., Mora, A., Morbidelli, R., Portell, J., Riva, A., Sarasso, M., Serraller, I., Siddiqui, H., Smart, R., Spagna, A., Stampa, U., Steele, I., Taris, F., Torra, J., van Reeven, W., Vecchiato, A., Zschocke, S., de Bruijne, J., Gracia, G., Raison, F., Lister, T., Marchant, J., Messineo, R., Soffel, M., Osorio, J., de Torres, A., O’Mullane, W. (2016). Gaia Data Release 1 - Astrometry: one billion positions, two million proper motions and parallaxes. Astronomy \& Astrophysics, 595, A4. https://doi.org/10.1051/0004-6361/201628714

Zariņš, A., Rubans, A., \& Silabriedis, G. (2016). Digital zenith camera of the University of Latvia. Geodesy and Cartography, 42, 129-135. https://doi.org/10.3846/20296991.2016.1268434 